\title{
Porcupine expression is associated with the expression of S100P and other cancer-related molecules in non-small cell lung carcinoma
}

\author{
BABETT BARTLING ${ }^{1}$, GRIT REHBEIN ${ }^{1}$, ANDREAS SIMM ${ }^{1}$, \\ ROLF-EDGAR SILBER ${ }^{1}$ and HANS-STEFAN HOFMANN ${ }^{2}$ \\ ${ }^{1}$ Department of Cardio-Thoracic Surgery, University Hospital Halle (Saale), Martin Luther University Halle-Wittenberg, \\ Halle (Saale); ${ }^{2}$ Department of Thoracic Surgery, Hospital Barmherzige Brüder Regensburg, Regensburg, Germany
}

Received October 30, 2009; Accepted December 21, 2009

DOI: 10.3892/ijo_00000582

\begin{abstract}
The o-acyltransferase Porcupine contributes to secretion and function of Wnt signaling molecules, which stimulate the expression of various cancer-related genes. Porcupine is also involved in the Wnt-induced cell signaling via B-Catenin in non-small cell lung carcinoma (NSCLC) cells. Herein, we report that the expression level of Porcupine in human NSCLC tissues $(n=89)$ positively correlates with the expression of several genes coding for cancer-related molecules such as $\beta$-Catenin, Hypoxia-inducible factor- $1 \alpha$ and Jun B. However, the mRNA expression of Porcupine was not generally increased in NSCLC compared to normal lung tissues. In NSCLC tissues we also found a positive correlation between the expression level of Porcupine and the calcium-binding protein S100P, which contributes to initiation and invasion of cancer cells. Subsequent studies showed that the DNA hypomethylation with 5-aza-2'deoxycytidine increased the mRNA expression of S100P in NSCLC cells but did not alter that of Porcupine. Silencing the expression of Porcupine with small interfering (si)RNA reduced the expression of S100P in NSCLC cells, whereas silencing the expression of S100P with siRNA did not effect the level of Porcupine expression. In conclusion, besides DNA methylation processes, Porcupine might regulate the expression of some cancer-related molecules including S100P in human NSCLC.
\end{abstract}

\section{Introduction}

Wnt [an amalgam of Wingless and Integration-1 (1)] glycoproteins are a family of secreted signaling molecules that control several signaling processes in embryos and in adults.

Correspondence to: Dr Babett Bartling, Klinik für Herz- und Thoraxchirurgie, Universitätsklinikum Halle (Saale), Ernst-GrubeStr. 40, D-61120 Halle (Saale), Germany

E-mail: babett.bartling@medizin.uni-halle.de

Key words: gene expression, microarray, non-small cell lung carcinoma, porcupine, S100P, ß-Catenin
Nineteen wnt genes are described in human, which additionally encode for alternatively spliced variants $(2,3)$. An altered regulation of Wnt proteins and/or compounds of their signaling pathway have also been linked to the development of a wide range of human cancers, in particular colorectal carcinomas $(3,4)$.

Wnt signaling requires Frizzled receptors and defined co-receptors at the cell surface that induces signaling either dependent on B-Catenin (canonical Wnt signaling) or independent of ß-Catenin (non-canonical Wnt signaling) (5). The canonical Wnt signaling is characterized by an increased level of cytoplasmic $\beta$-Catenin due to protecting it from the proteasome degradation machinery $(6,7)$. The consequence of stabilized B-Catenin is its increased nuclear translocation, complex formation with the Lef (lymphoid enhancer factor)1/ Tcf (T cell-factor) transcription factors and expression of target genes such as the proto-oncogene myc (8). The canonical Wnt signaling can be antagonized by the non-canonical Wnt signaling, which involves intracellular calcium release, activation of Rho GTPases and JNK (c-Jun N-terminal kinase) $(5,9)$. Despite of differences to the colorectal carcinoma, both types of Wnt-induced cell signaling have emerged as critical pathways in lung carcinogenesis (10).

Wnt proteins are secreted from the cell by the endoplasmic reticulum (ER)-Golgi classical pathway (11). For this process, they are modified due to acylation by palmitoyl and palmitoleoyl groups (12-14). The putative enzyme for this lipid modification of Wnt proteins is the ER-resident o-acyltransferase Porcupine (13-16), also called MG61 in human (17). Wnt acylation by Porcupine supports the $N$-glycosylation of Wnt proteins at the ER membrane, which is necessary for the Wnt secretion via Golgi $(18,19)$. Moreover, Wnt acylation contributes to the binding of Wnt to its surface receptors and, therefore, Wnt-induced cell signaling $(12,20)$.

It has been reported that the transcription of Porcupine is increased in the non-small cell lung carcinoma (NSCLC) (21), which composes about $80 \%$ of all lung malignancies (22). Silencing the expression of Porcupine in selected NSCLC cell lines resulted in a strong down-regulation of $\beta$ Catenin and its targeted gene products (cyclin D1, dishevelled, frizzled 7 , survivin) as well as in a dramatically increased apoptotic cell death (21). These data suggest the 
contribution of Porcupine to the canonical Wnt signaling, and probably non-canonical Wnt signaling, in NSCLC development. Because the Wnt-induced cell signaling stimulates the expression of various cancer-related genes (2), some gene expressions might also be regulated by the level of Porcupine. Therefore, our study focused on the level of Porcupine expression and its relation to the expression of cancer-related genes in human NSCLC tissues and cells.

\section{Materials and methods}

NSCLC tissues and cells. We studied NSCLC tissues and non-involved normal lung tissues of lung cancer patients, who underwent pulmonary resection surgery. Only patients with clear histological classification as NSCLC (adenocarcinoma, squamous cell carcinoma) and without neoadjuvant chemo- or radiotherapy were admitted to the study. Immediately after resection the lung tissues were snap-frozen and stored in liquid nitrogen until use. The local ethics committee approved this study and the subjects gave informed consent.

Human NSCLC cells used in this study were the National Cancer Institute (NCI-) cell lines A549, H358 and H322 (ATCC; Manassas, VA, USA). Cells were cultured in DMEM supplemented with $10 \%$ fetal calf serum in a humidified atmosphere at $10 \% \mathrm{CO}_{2}$ and $37^{\circ} \mathrm{C}$. Number and viability of cells were estimated after counting the total number of living and death cells using the Casy ${ }^{\circledR}$ cell counter (Schärfe System $\mathrm{GmbH}$; Reutlingen, Germany). DNA hypomethylation was induced by treating cells with $10 \mu \mathrm{M}$ 5-aza-2'deoxycytidine (Sigma; Taufkirchen, Germany). Silencing the expression of human Porcupine or S100P was induced by transient transfection of 100 pmol synthetic small interfering (si)RNA using Oligofectamine ${ }^{\mathrm{TM}}$ reagent (Invitrogen; Carlsbad, CA, USA). The targeted sequence of the siRNA duplex was 5'-A AGTTGTCACAAGCTGGAACC-3' for Porcupine (21), 5'-A ATGGAGATGCCCAGGTGGAC-3' for S100P (23) and 5'-A ATGAGGATGCCTGCAGGGAC-3' for control (scrambled siRNA; Invitrogen). S100P overexpression was induced by transient transfection of the coding sequence of human S100P, which had been cloned into the pcDNA3.1(+) expression vector (Invitrogen), by the use of Effectene ${ }^{\circledR}$ reagent (Qiagen; Hilden, Germany). Control transfection was performed with empty pcDNA3.1(+) vector.

RNA analysis. RNA was isolated with TRIzol ${ }^{\circledR}$ reagent (Invitrogen) and/or RNeasy Mini kit (Qiagen). For mRNA expression analysis by polymerase chain reaction (PCR) RNA was transcribed to cDNA with Superscript II ${ }^{\mathrm{TM}}$ reverse transcriptase (Invitrogen). Thereafter, cDNA was amplified by real-time PCR containing cDNA-specific primer pairs (Biomers; Ulm, Germany) and SYBR green PCR mix (BioRad; Munich, Germany). We used cDNA-specific primers for PCR amplification of human Porcupine [sense: 5'-TAC CTGAAGCATGCAAGCAC-3', antisense: 5'-CGGTGTCTA CCATGTGCATC-3' (21)], S100P [sense: 5'-ATCTAGCAC CATGACGGAAC-3', antisense: 5'-AGCCTAGGGGAATAA TTGCC-3' (24)] and 18S rRNA [sense: 5'-GTTGGTGGAG CGATTTGTCTGG-3', antisense: 5'-AGGGCAGGGACTT AATCAACGC-3' (25)]. External cDNA standards with identical primer-binding sites were established for PCR amplification of a concentration series of standard molecules. Real-time PCR was performed using the iCycler $\mathrm{iQ}^{\mathrm{TM}}$ system (Bio-Rad; Hercules, CA, USA). Calculating the signal for Porcupine or S100P in relation to the signal for $18 \mathrm{~S}$ rRNA assessed the relative gene expression. Standard PCR was performed using the 2X PCR mix (Promega; Mannheim, Germany). After PCR amplification in a thermocycler (Biometra; Göttingen, Germany) and subsequent agarose gelelectrophoresis, PCR products were estimated by use of the LAS 3000 computer-based imaging system (FujiFilm; Tokyo, Japan).

For mRNA expression analysis by high-density oligonucleotide microarray (Affymetrix; Santa Clara, CA, USA), biotinylated target cRNA was prepared from total RNA as described (26) and then hybridized to the microarrays according to Affymetrix GeneChip technology (27). Microarrays were washed and stained with the GeneChip Fluidics Station 400. The intensity of the hybridization signals was scanned with the GeneArray Scanner $7 \mathrm{G}$ and analyzed by using the GeneChip ${ }^{\circledR}$ Operating System.

Protein analysis. Total protein was extracted from cells in Tris(hydroxymethyl)amino-methane-buffered sodium dodecyl sulfate solution containing protease inhibitors and subjected to standard immunoblot procedure. Mouse monoclonal antiB-Catenin (BD Bioscience; Heidelberg, Germany) and goat polyclonal anti-S100P (R\&D Systems; Minneapolis, MN, USA) were used as primary antibodies. Specificity of the antiS100P was checked by use of protein extracts from S100Poverexpressing cells (24). GAPDH immunoblotting with a rabbit polyclonal antibody (Abcam; Cambridge, UK) indicated protein loading. Primary antibodies were visualized by horseradish peroxidase-conjugated secondary antibodies and ECLplus detection (Amersham; Buckinghamshire, UK). The signal intensities were analyzed by use of the LAS 3000 computer-based imaging system (FujiFilm) equipped with AIDA 3.5. software (Raytest; Straubenhardt, Germany).

Data analysis. Patient's data were calculated as median with interquartile range. Experimental data were calculated as mean \pm standard error. Statistical significance of the data $(\mathrm{P}<0.05)$ was determined by Student's t-test followed by Mann-Whitney rank sum test or by the ANOVA procedure followed by Dunnett's method as appropriated (SigmaStat 3.5 software; Jandel Corp.; San Rafael, CA, USA). The correlation coefficient $\mathrm{R}$ of the linear regression analysis for significance was tested using the two-sided test.

\section{Results}

Porcupine expression in NSCLC. Oligonucleotide microarray analysis of human NSCLC tissues revealed a highly significant correlation of the mRNA expression level of many genes with that of Porcupine. While the expression level of 791 genes positively correlated with the mRNA expression of Porcupine $(n=89, R>0.350)$, the expression level of only 2 genes showed a negative correlation $(\mathrm{R}<-0.350)$. Among the expressions correlating positively with the mRNA expression level of Porcupine we found the expression of some genes coding for 
Table I. Selected gene products whose expression correlates with the Porcupine expression in NSCLC tissue.

\begin{tabular}{|c|c|c|c|c|c|c|}
\hline \multirow[b]{2}{*}{ Gene product } & \multirow{2}{*}{$\begin{array}{l}\text { GenBank } \\
\text { accession }\end{array}$} & \multicolumn{2}{|c|}{$\begin{array}{c}\text { Correlation with } \\
\text { Porcupine expression }\end{array}$} & \multicolumn{3}{|c|}{ Median expression } \\
\hline & & Coefficient R & P-value & NSCLC & Normal lung & P-value \\
\hline \multicolumn{7}{|l|}{ Positive correlation } \\
\hline E (epithelial)-Cadherin & NM_004360 & 0.371 & 0.0002 & 777 & 631 & 0.0153 \\
\hline$\alpha$-Catenin & NM_001903 & 0.414 & $<0.0001$ & 371 & 379 & 0.9916 \\
\hline ß-Catenin & BE178050 & 0.410 & $<0.0001$ & 369 & 385 & 0.4377 \\
\hline$\delta$-Catenin & NM_001331 & 0.448 & $<0.0001$ & 306 & 308 & 0.3735 \\
\hline Cathepsin D & AI565004 & 0.469 & $<0.0001$ & 674 & 452 & $<0.0001$ \\
\hline Endothelin-1 & NM_001955 & 0.466 & $<0.0001$ & 137 & 250 & $<0.0001$ \\
\hline Ezrin (Villin 2) & NM_003379 & 0.501 & $<0.0001$ & 706 & 818 & 0.0007 \\
\hline Hypoxia-inducible factor (HIF)- $1 \alpha$ & NM_001530 & 0.443 & $<0.0001$ & 548 & 462 & 0.0032 \\
\hline c-Jun N-terminal kinase (JNK) 1 & AA257992 & 0.414 & $<0.0001$ & 703 & 582 & $<0.0001$ \\
\hline Jun B transcription factor & NM_002229 & 0.467 & $<0.0001$ & 919 & 784 & 0.0002 \\
\hline Plakophilin-3 & NM_007183 & 0.363 & 0.0003 & 501 & 296 & $<0.0001$ \\
\hline Plakophilin-4 & AL031846 & 0.374 & 0.0002 & 251 & 509 & $<0.0001$ \\
\hline Rho family small GTP binding protein Rac1 & NM_006908 & 0.471 & $<0.0001$ & 761 & 727 & 0.5053 \\
\hline Rho GTPase activating protein 1 & NM_004308 & 0.415 & $<0.0001$ & 678 & 701 & 0.6598 \\
\hline S100 calcium-binding protein A6 (S100A6) & NM_014624 & 0.504 & $<0.0001$ & 918 & 1068 & 0.0096 \\
\hline S100 calcium-binding protein P (S100P) & NM_005980 & 0.457 & $<0.0001$ & 395 & 206 & 0.0050 \\
\hline \multicolumn{7}{|l|}{ Negative correlation } \\
\hline ING1-like tumor suppressor protein & AF149724 & -0.382 & 0.0013 & 161 & 161 & 0.8530 \\
\hline Solute carrier family 17 member 7 & NM_020309 & -0.358 & 0.0037 & 274 & 279 & 0.8679 \\
\hline
\end{tabular}

mRNA expression was determined for $n=89$ NSCLC tissues (study group 1) by oligonucleotide microarray analysis, and the expression level is given as normalized microarray signal intensity. GenBank accession of Porcupine: NM_022825. Exclusion criteria were low expression level (normalized microarray signal intensity $<150)$ and low correlation coefficient of the linear regression $(-0.350<\mathrm{R}<+0.350)$.

cancer-related molecules such as $\beta$-Catenin, HIF-1 $\alpha$ and Jun B (for further examples see Table I). In the case of Wnt/B-Catenin target gene products (2) we only found a significant correlation between the mRNA expression level of Porcupine with that of E-Cadherin and Endothelin-1 (Table I).

Some gene products listed in Table I also showed an altered mRNA expression in NSCLC compared with non-involved normal lung tissue such as S100P and its intracellular interaction partner Ezrin. While the mRNA expression of S100P was increased in NSCLC compared with normal lung tissue, the expression of Erzin was reduced in NSCLC (Table I). In contrast, the mRNA expression of Porcupine was not significantly altered in NSCLC compared with normal lung tissue despite of its well detectable expression in NSCLC tissues and cell lines (Fig. 1, Table II). This has been shown for two independent study groups analyzed by either oligonucleotide microarray or real-time PCR (Table II). In both study groups the number of normal lung tissues was lower than the number of NSCLCs, because we only selected normal lung tissues without signs of pathologies. However, paired analyses of NSCLC and normal lung tissue of the same patient did also not reveal significant changes in the mRNA expression (Table II).

Porcupine regulates the S100P expression in NSCLC cells. $\mathrm{S} 100 \mathrm{P}$ is reported as gene product involved in the initiation and invasion of cancer cells $(23,24,28-35)$ as well as in the survival of cancer patients $(30,33)$. Therefore, our further analyses focused on the interaction between the expression level of Porcupine and S100P in NSCLC. First of all, we studied the impact of DNA hypomethylation by 5-aza-2' deoxycytidine on the expression of Porcupine and S100P in the NSCLC cell line NCI-H358. This deoxycytidine analogue increased the mRNA expression of S100P but not Porcupine after 2 to 3 days (Fig. 2). We then checked whether the DNA hypomethylation-induced increase in S100P might have simultaneously impaired the mRNA expression of Porcupine. However, transient overexpression of S100P in NCI-H358 did not change the expression level of Porcupine compared with control transfected cells after 2 to 3 days (data not shown).

Finally, we studied the effect of Porcupine silencing on the expression of S100P in NCI-H358 and further NSCLC cell lines (NCI-H322 and NCI-A549) by siRNA transfection. The transient transfection of NCI-H358 cells with Porcupinetargeted siRNA reduced the mRNA expression of S100P for 1 to 2 days (Fig. 3A, left panel). This effect was even more pronounced in the cell line NCI-H322 but less in NCI-A549 (Fig. 3A, left panel). The reduced expression of S100P following Porcupine-targeted siRNA transfection was also determined on protein level in the NSCLC cell lines (Fig. 3B), but extent and time of the reduction of S100P protein slightly 
NSCLC tissues

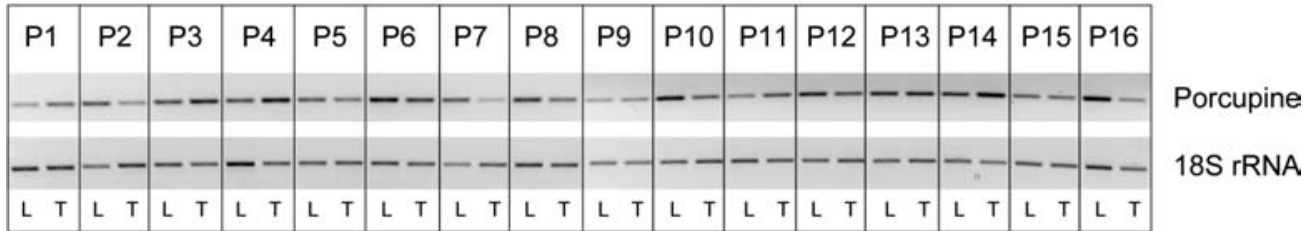

NSCLC cell lines

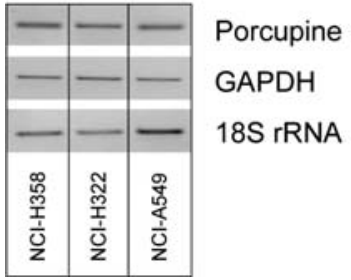

Figure 1. mRNA expression of Porcupine is shown after PCR amplification for NSCLC tissue and paired normal lung tissue of the same patient (corresponding to study group 2 in Table II; gel loading of the real-time PCR) as well as for human NSCLC cell lines (gel loading of the standard PCR). Amplification of $18 \mathrm{~S}$ rRNA and/or GAPDH indicates PCR loading.

Table II. Porcupine expression in NSCLC tissue.

Study group

1: All tissues

Number

Porcupine expression ${ }^{\mathrm{a}}$

1: Paired tissues only

Number

Porcupine expression ${ }^{a}$

2: All tissues

Number

Porcupine expression ${ }^{b}$

2: Paired tissues only

Number

Porcupine expression ${ }^{\mathrm{b}}$

NSCLC

0.267 (0.182-0.484)

16

$0.263(0.201-0.525)$
Normal lung

464 (430-485)

16

$0.246(0.146-0.340)$

16

$0.246(0.146-0.340)$

0.090

mRNA expression level was determined by aligonucleotide microarray analysis (normalized microarray signal intensity) and ${ }^{\mathrm{b}}$ real time-PCR (normalized per 18S rRNA signal intensity). Expression data are given as median with interquartile range.

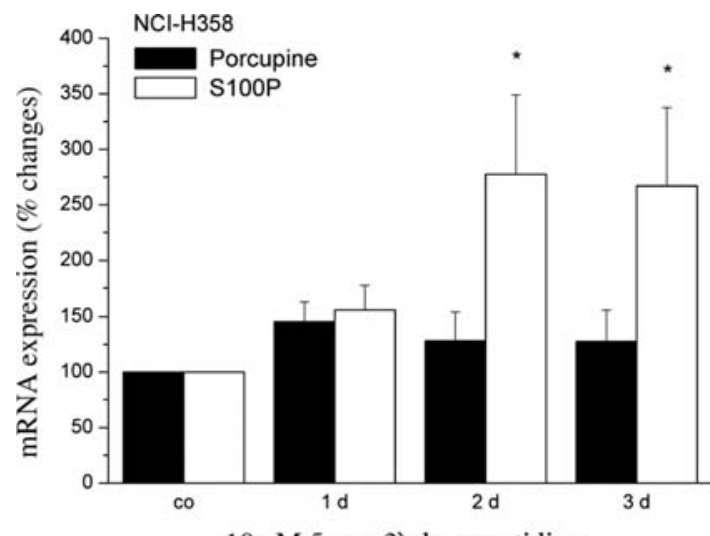

\section{$\mathrm{NCl}-\mathrm{H} 358$}

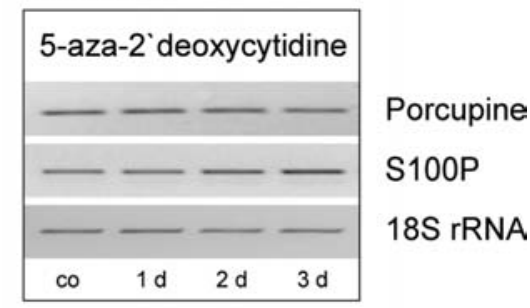

$10 \mu \mathrm{M} 5$-aza-2` deoxycytidine

Figure 2. Time-dependent effect of 5-aza-2'deoxycytidine on the mRNA expression of Porcupine and S100P in the NSCLC cell line NCI-H358. mRNA expression was determined by PCR amplification per $18 \mathrm{~S}$ rRNA as loading control. Mean data are given \pm standard error $(\mathrm{n}=6)$ with ${ }^{*} \mathrm{P}<0.05$ vs. untreated control (co). 
A
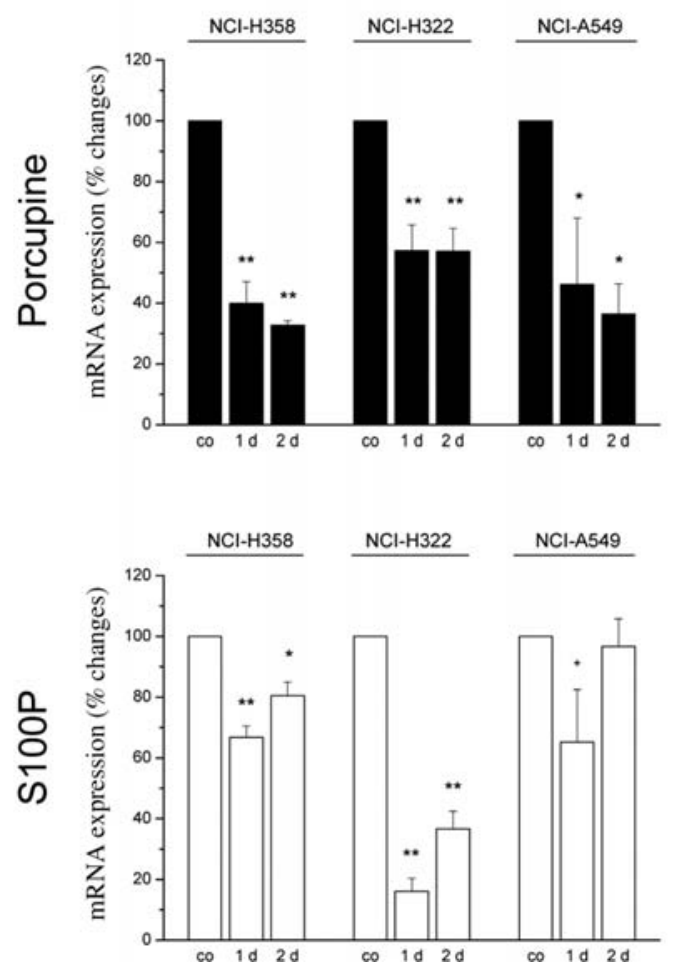

B

Porcupine siRNA
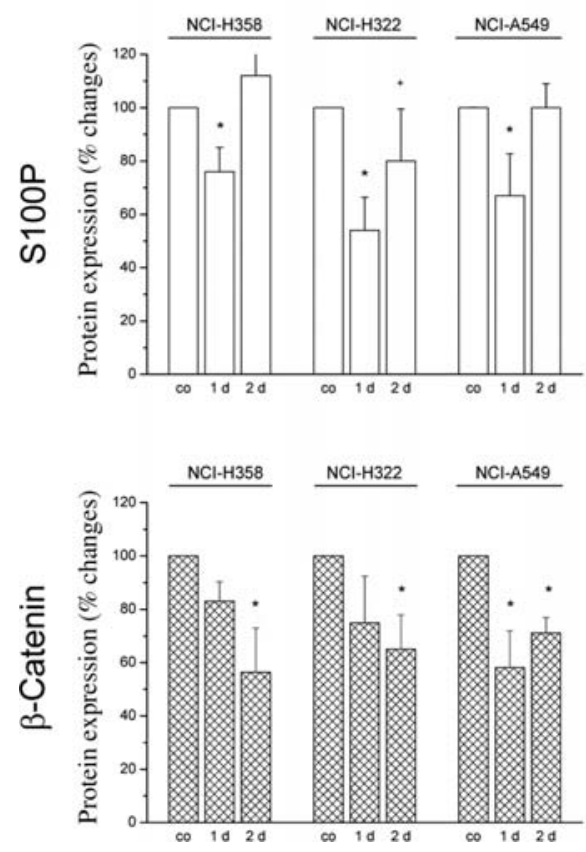

S100P siRNA
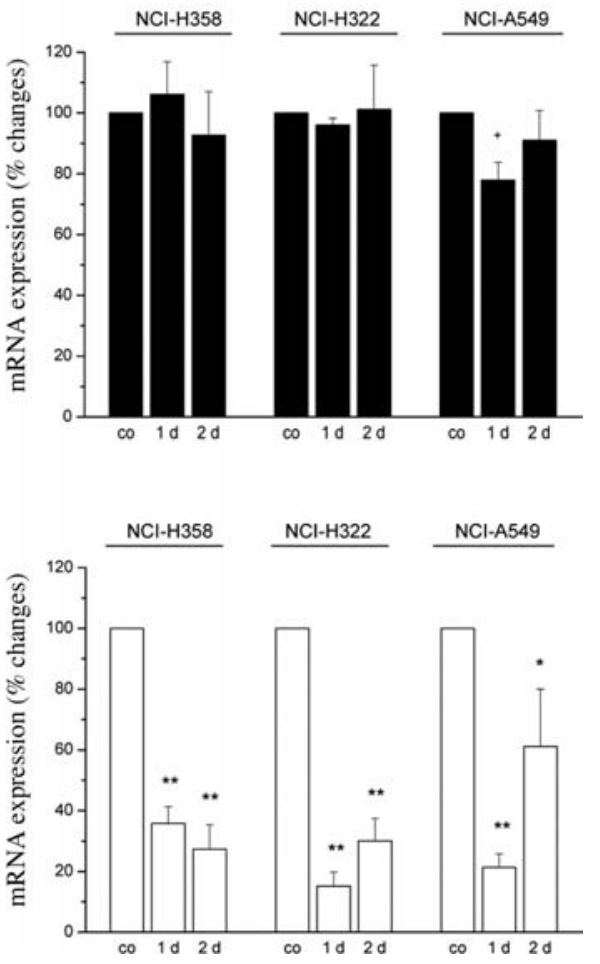

$\mathrm{NCl}-\mathrm{H} 358$

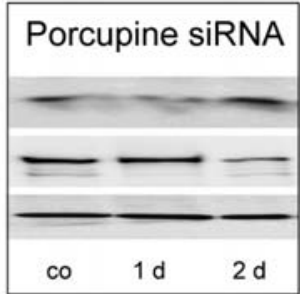

S100P (11 kDa)

$\beta$-Catenin (94 kDa)

GAPDH (36 kDa)

Figure 3. Time-dependent effect of the transfection with Porcupine- or S100P-targeted siRNA on the (A) mRNA expression of Porcupine and S100P and (B) protein expression of S100P and B-Catenin in the NSCLC cell lines NCI-H358, NCI-H322 and NCI-A549. (B) mRNA expression was determined by PCR amplification per 18S rRNA as loading control and protein expression was determined by immunoblot detection per GAPDH as loading control. Immunoblot detection of S100P and B-Catenin is exemplarily shown for NCI-H358 cells. Mean data are given \pm standard error ( $\mathrm{n} \geq 3$ ) with ${ }^{+} \mathrm{P}=0.1$, ${ }^{*} \mathrm{P}<0.05$ and ${ }^{* *} \mathrm{P}<0.01$ vs. control transfection with scrambled siRNA (co).

differed from the reduction of S100P mRNA (Fig. 3A). Extent and time of the protein reduction of S100P also differed from the reduction of $\beta$-Catenin protein following transfection of
Porcupine-targeted siRNA (Fig. 3B). Conversely, the siRNAmediated silencing of S100P did not change the expression level of Porcupine in the NSCLC cell lines (Fig. 3A, right 
panel). The viability of the cell lines was not obviously impaired by the Porcupine- or S100P-targeted siRNA ( $\geq 90 \%$ viable cells at day 1 to 3 after siRNA transfection).

\section{Discussion}

Various cancer-related genes are activated by Wnt signaling, in which the o-acyltransferase Porcupine plays an important role $(4,36)$. Our study showed that the expression of Porcupine positively correlates with the expression of several genes coding for cancer-related molecules in human NSCLC tissues. Among them we identified the calcium-binding protein S100P, which is a member of the S100 family (37). Subsequent studies revealed that the expression of S100P in NSCLC cells can be regulated by Porcupine in addition to DNA methylation.

S100P has been suggested as a gene product responsible for the immortalization of epithelial cells that finally leads to neoplastic transformation $(28,29,31)$. Although the exact contribution of S100P to this process is unclear, S100P is increased in early stages of human NSCLC $(30,38)$ and other carcinomas (28,39-41). In addition, the increase in S100P has been revealed as a predictor of worse outcome of NSCLC patients (30). This clinical observation is explained by the supporting effect of S100P on cancer cell invasion in vitro and in vivo $(23,30,32-35)$. One of the molecular mechanisms responsible for the increased expression of S100P in cancer cells is the DNA hypomethylation in $5^{\prime} \mathrm{CpG}$ islands of the s100P gene as identified for pancreatic and prostate cancer $(42,43)$ and by our group for lung cancer $(24)$. Although this indicates the importance of DNA methylation processes in the transcriptional regulation of S100P, our siRNA studies showed that the expression of S100P is also regulated by Porcupine in NSCLC cells. However, NSCLC cells seem to have a different turnover of S100P mRNA and protein because the expression of S100P differed at mRNA and protein level following siRNA-mediated silencing of Porcupine. In contrast to S100P, the transcription of Porcupine was independent of DNA hypomethylation. Feed-back mechanisms of S100P on the transcription of Porcupine can be excluded because silencing or overexpression of S100P did not influence the mRNA level of Porcupine.

The lipid modification of Wnt proteins by Porcupine is required for the Wnt processing $(11,36)$. Therefore, Porcupine might rather stimulate than suppress the transcription of various genes via Wnt pathways. This assumption is supported by our observation that the expression level of Porcupine in NSCLC tissues correlates positively with that of others genes in nearly all cases. Among these genes we found some genes coding for cancer-related molecules such as the transcription factors HIF- $1 \alpha$ and Jun B, which themselves activate the expression of cancer-related genes $(44,45)$ as well as Cathepsin D and Ezrin, which are related to the function of S100P in cancer cells $(34,35)$. In addition, we observed a positive correlation between the expression level of Porcupine and B-Catenin in NSCLC tissues. In accordance with this observation, silencing Porcupine induced a diminished expression of $B$-Catenin in NSCLC cells. This effect of Porcupine on the expression of $\beta$-Catenin and the $\beta$-Catenin-dependent (canonical) Wnt signaling pathway in NSCLC cells was shown recently (21). As human NSCLC cells express the canonical Wnt molecules Wnt2, Wnt3, Wnt7a and Wnt9a (46), it suggests the primary contribution of Porcupine to the canonical Wnt signaling in NSCLC.

However, cancer development highly depends on the tumor stroma that can be a source of the non-canonical Wnt molecules (Wnt 4, Wnt5a and Wnt11) $(9,10,47)$. The possible involvement of Porcupine in the non-canonical Wnt signaling is supported by our observation that the mRNA expression of only few target gene products of $\beta$-Catenin (E-Cadherin, Endothelin-1) correlates with that of Porcupine in NSCLC tissues. Moreover, transient silencing of Porcupine by siRNA induced a less stable reduction of S100P compared to B-Catenin in NSCLC cells. Altogether, these data indicate the contribution of Porcupine to canonical and non-canonical Wnt signaling pathways in NSCLC.

Porcupine has been suggested as a critical factor involved in the survival of NSCLC cells but not lung fibroblasts by attenuating the canonical Wnt pathway (21). Although we used the same NSCLC cell line (NCI-A549), transient silencing of Porcupine by siRNA did not affect the viability of the NSCLC cells. However, it is possible that we did not achieve that long-term reduction of Porcupine/ß-Catenin necessary for inducing cell death. In this regard, it also has to remember that $B$-Catenin is a molecule with dual function. It is involved in both canonical Wnt signaling (6) and epithelial cell-cell adhesion via intracellular binding to E-Cadherin in complex with $\alpha$-Catenin (48). The expression level of E-Cadherin and $\alpha$-Catenin, which are often dysfunctional in lung carcinomas due to mutations (48), also correlated positively with that of Porcupine in NSCLC tissues. Thus, Porcupine might play an important role in NSCLC as well as in normal lung epithelium but at different molecular level.

Differential gene expressions between tumor and nontumor tissue do not necessarily indicate the contribution of individual gene products to tumor development. In particular, the dissimilar cellular composition of tumor and non-tumor tissue may strongly influence the expression level of total tissues. In this regard, we did not find a general increase in the mRNA expression level of Porcupine in human NSCLC compared to normal lung tissues contrary to recent reports (21). This has been shown for a high number of NSCLC tissues in two study groups and for two analytical methods. An assessment of the protein expression of Porcupine is presently not possible because of unavailable antibodies. However, the direct correlation of the expression level of Porcupine with the transcription of some cancer-related genes in human NSCLC tissues suggests the gene-regulatory role of Porcupine in NSCLC. Although the correlation of gene expressions in tissue samples can not point out the molecular interaction of gene products in single cells, our experimental studies indicated that Porcupine regulates the expression of S100P in NSCLC additionally to DNA methylation.

In conclusion, Porcupine might contribute to NSCLC development by transcriptional activation of cancer-related genes such as $s 100 P$.

\section{Acknowledgements}

We thank Professor G. Hansen (Hannover Medical School, Germany) and Professor S. Burdach (Technical University 
Munich, Germany) for cooperation with the microarray analysis. This project was supported by the Deutsche Krebshilfe (107078), EOS Biotechnology (Fremont, CA, USA) and Wilhelm Roux program of the Bundesministerium für Bildung und Forschung (FKZ 14/14, FKZ 3/30). The sponsors of the study had no role in data interpretation or writing of the report.

\section{References}

1. Nusse R, Brown A, Papkoff J, et al: A new nomenclature for int-1 and related genes: the Wnt gene family. Cell 64: 231, 1991.

2. http://wwwstanfordedu/\% 7ernusse/pathways/targetshtml.

3. Klaus A and Birchmeier W: Wnt signaling and its impact on development and cancer. Nat Rev Cancer 8: 387-398, 2008.

4. Paul S and Dey A: Wnt signaling and cancer development: therapeutic implication. Neoplasma 55: 165-176, 2008.

5. Widelitz R: Wnt signaling through canonical and non-canonical pathways: recent progress. Growth Factors 23: 111-116, 2005.

6. Clevers H: Wnt/beta-catenin signaling in development and disease. Cell 127: 469-480, 2006

7. Huang $\mathrm{H}$ and $\mathrm{He} \mathrm{X}$ : Wnt/beta-catenin signaling: new (and old) players and new insights. Curr Opin Cell Biol 20: 119-125, 2008.

8. He TC, Sparks AB, Rago C, et al: Identification of c-MYC as a target of the APC pathway. Science 281: 1509-1512, 1998.

9. Veeman MT, Axelrod JD and Moon RT: A second canon. Functions and mechanisms of beta-catenin-independent Wnt signaling. Dev Cell 5: 367-377, 2003.

10. Mazieres J, He B, You L, et al: Wnt signaling in lung cancer. Cancer Lett 222: 1-10, 2005.

11. Bartscherer K and Boutros M: Regulation of Wnt protein secretion and its role in gradient formation. EMBO Rep 9: 977-982, 2008

12. Willert K, Brown JD, Danenberg E, et al: Wnt proteins are 202 lipid-modified and can act as stem cell growth factors. Nature 423: 448-452, 2003.

13. Zhai L, Chaturvedi D and Cumberledge S: Drosophila wnt-1 undergoes a hydrophobic modification and is targeted to lipid rafts, a process that requires porcupine. J Biol Chem 279: 33220-33227, 2004

14. Takada R, Satomi Y, Kurata T, et al: Monounsaturated fatty acid modification of Wnt protein: its role in Wnt secretion. Dev Cell 11: 791-801, 2006.

15. Kadowaki T, Wilder E, Klingensmith J, et al: The segment polarity gene porcupine encodes a putative multitransmembrane protein involved in Wingless processing. Genes Dev 10: 3116-3128, 1996.

16. Hofmann K: A superfamily of membrane-bound O-acyltransferases with implications for wnt signaling. Trends Biochem Sci 25: $111-112,2000$.

17. Caricasole A, Ferraro T, Rimland JM and Terstappen GC: Molecular cloning and initial characterization of the MG61/ PORC gene, the human homologue of the Drosophila segment polarity gene Porcupine. Gene 288: 147-157, 2002.

18. Tanaka K, Okabayashi K, Asashima M, et al: The evolutionarily conserved porcupine gene family is involved in the processing of the Wnt family. Eur J Biochem 267: 4300-4311, 2000.

19. Tanaka K, Kitagawa Y and Kadowaki T: Drosophila segment polarity gene product porcupine stimulates the posttranslational $\mathrm{N}$-glycosylation of wingless in the endoplasmic reticulum. J Biol Chem 277: 12816-12823, 2002.

20. Komekado H, Yamamoto H, Chiba T and Kikuchi A: Glycosylation and palmitoylation of Wnt-3a are coupled to produce an active form of Wnt-3a. Genes Cells 12: 521-534, 2007.

21. Chen Z, Li J, Li QS, et al: Suppression of PPN/MG61 attenuates Wnt/beta-catenin signaling pathway and induces apoptosis in human lung cancer. Oncogene 27: 3483-3488, 2008.

22. Beasley MB, Brambilla E and Travis WD: The 2004 World Health Organization classification of lung tumors. Semin Roentgenol 40: 90-97, 2005.

23. Arumugam T, Simeone DM, Van Golen K and Logsdon CD S100P promotes pancreatic cancer growth, survival, and invasion. Clin Cancer Res 11: 5356-5364, 2005.

24. Rehbein G, Simm A, Hofmann H, et al: Molecular regulation of S100P in human lung adenocarcinomas. Int J Mol Med 22: $69-77,2008$.
25. Hofmann H, Simm A, Hammer A, et al: Expression of inhibitors of apoptosis (IAP) proteins in non-small cell human lung cancer. J Cancer Res Clin Oncol 128: 554-560, 2002.

26. Golub T, Slonim D, Tamayo P, et al: Molecular classification of cancer: class discovery and class prediction by gene expression monitoring. Science 286: 531-537, 1999.

27. Lipshutz R, Fodor S, Gingeras T and Lockhart D: High density synthetic oligonucleotide arrays. Nat Genet 21: 20-24, 1999.

28. Guerreiro Da Silva I, Hu Y, Russo I, et al: S100P calciumbinding protein overexpression is associated with immortalization of human breast epithelial cells in vitro and early stages of breast cancer development in vivo. Int J Oncol 16: 231-241, 2000.

29. Hellung Schonning B, Bevort M, Mikkelsen S, et al: Human papillomavirus type 16 E7-regulated genes: regulation of S100P and ADP/ATP carrier protein genes identified by differentialdisplay technology. J Gen Virol 81: 1009-1015, 2000.

30. Diederichs S, Bulk E, Steffen B, et al: S100 family members and trypsinogens are predictors of distant metastasis and survival in early-stage non-small cell lung cancer. Cancer Res 64: 5564-5569, 2004.

31. Gibadulinova A, Barathova M, Kopacek J, et al: Expression of S100P protein correlates with and contributes to the tumorigenic capacity of HeLa cervical carcinoma cells. Oncol Rep 14: 575-582, 2005 .

32. Arumugam T, Ramachandran V and Logsdon CD: Effect of cromolyn on S100P interactions with RAGE and pancreatic cancer growth and invasion in mouse models. J Natl Cancer Inst 98: 1806-1818, 2006.

33. Wang G, Platt-Higgins A, Carroll J, et al: Induction of metastasis by $\mathrm{S} 100 \mathrm{P}$ in a rat mammary model and its association with poor survival of breast cancer patients. Cancer Res 66: 1199-1207, 2006

34. Whiteman HJ, Weeks ME, Dowen SE, et al: The role of S100P in the invasion of pancreatic cancer cells is mediated through cytoskeletal changes and regulation of cathepsin D. Cancer Res 67: 8633-8642, 2007.

35. Austermann J, Nazmi AR, Muller-Tidow C and Gerke V: Characterization of the $\mathrm{Ca}^{2+}$-regulated ezrin-S100P interaction and its role in tumor cell migration. J Biol Chem 283: 29331-29340, 2008.

36. Mikels AJ and Nusse R: Wnts as ligands: processing, secretion and reception. Oncogene 25: 7461-7468, 2006.

37. Becker T, Gerke V, Kube E and Weber K: S100P, a novel $\mathrm{Ca}(2+)$-binding protein from human placenta. cDNA cloning, recombinant protein expression and $\mathrm{Ca}^{2+}$ binding properties. Eur J Biochem 207: 541-547, 1992.

38. Bartling B, Rehbein G, Schmitt W, et al: S100A2-S100P expression profile and diagnosis of non-small cell lung carcinoma: impairment by advanced tumour stages and neoadjuvant chemotherapy. Eur J Cancer 43: 1935-1943, 2007.

39. Dowen SE, Crnogorac-Jurcevic T, Gangeswaran R, et al: Expression of S100P and its novel binding partner S100PBPR in early pancreatic cancer. Am J Pathol 166: 81-92, 2005.

40. Ohuchida K, Mizumoto K, Egami T, et al: S100P is an early developmental marker of pancreatic carcinogenesis. Clin Cancer Res 12: 5411-5416, 2006.

41. Schor AP, Carvalho FM, Kemp C, et al: S100P calcium-binding protein expression is associated with high-risk proliferative lesions of the breast. Oncol Rep 15: 3-6, 2006.

42. Sato N, Fukushima N, Matsubayashi H and Goggins M: Identification of maspin and S100P as novel hypomethylation targets in pancreatic cancer using global gene expression profiling. Oncogene 23: 1531-1538, 2004.

43. Wang Q, Williamson M, Bott S, et al: Hypomethylation of WNT5A, CRIP1 and S100P in prostate cancer. Oncogene 26: 6560-6565, 2007.

44. Vogt PK: Jun, the oncoprotein. Oncogene 20: 2365-2377, 2001.

45. Semenza GL: Targeting HIF-1 for cancer therapy. Nat Rev Cancer 3: 721-732, 2003 .

46. Akiri G, Cherian MM, Vijayakumar S, et al: Wnt pathway aberrations including autocrine Wnt activation occur at high frequency in human non-small-cell lung carcinoma. Oncogene 28: 2163-2172, 2009.

47. Macheda ML and Stacker SA: Importance of Wnt signaling in the tumor stroma microenvironment. Curr Cancer Drug Targets 8: 454-465, 2008

48. Hirohashi S and Kanai Y: Cell adhesion system and human cancer morphogenesis. Cancer Sci 94: 575-581, 2003. 